\title{
Interim view from NASA relativity probe
}

Gravity Probe B (GP-B) seems like the experiment that never ends. The spacecraft set records for the longest-running development project at NASA -40 years - and data analysis is stretching into its second year. The GP-B team may one day announce the most precise measurements yet of a long-sought effect of general relativity. But that didn't happen on 14 April, when project scientists presented an interim report to the American Physical Society meeting in Jacksonville, Florida.

Team members promise a final report by December, when money for the \$760-million experiment runs out. But it is clear that unexpected systematic errors will make it a real challenge to reach the original mission goals. For the most subtle effect measured, the GP-B team needs to make the experiment's uncertainty 100 times lower.

GP-B is a simple concept that in reality proved overwhelming. The experiment, proposed in 1964, required four perfect spinning gyroscopes in Earth orbit to measure how the spinning planet drags the fabric of space-time around with it - a phenomenon predicted by Einstein's general theory of relativity

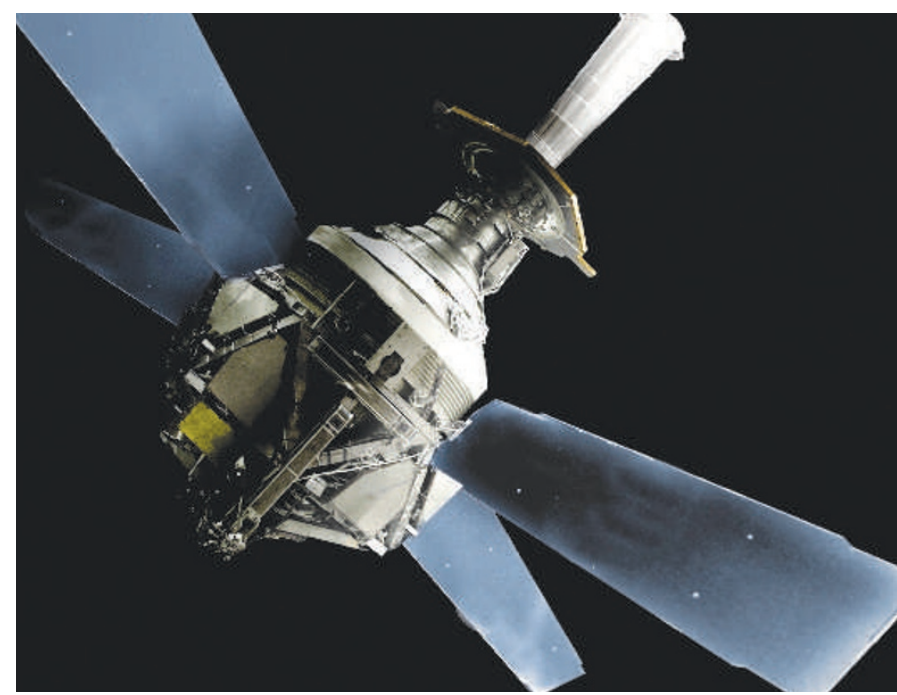

NASA's Gravity Probe B aims to confirm two predictions of general relativity.

and called 'frame-dragging'. The mission survived every NASA attempt to cancel it (see Nature 426, $380-381 ; 2003$ ) and was finally launched in April 2004.

The main goal was to measure frame-dragging to within $1 \%$ accuracy. That would be ten times better than the best measurements so far, which were taken by bouncing laser signals off the Earthorbiting LAGEOS satellites.

GP-B has had one success. At the meeting, the team announced the first experimental measurement of geodetic precession, another small distortion of space-time. The geodetic precession of the Moon around the Earth has previously been verified to an accuracy of about $0.7 \%$. GP-B has now measured the effect on their probe to within $1.5 \%$. The team hopes to reduce this further - but it is the much smaller frame-dragging effect that everyone cares about.

The problems plaguing the analysis are systematic errors: electrostatic effects on the spheres at the core of the gyroscopes cause misalignments and wobbles that vary unexpectedly over time. The data were also recorded in chunks because solar flares required the system to be rebooted.

Physicist Clifford Will, who chairs NASA's scientific advisory committee for the project, says such errors are a real headache: “There's art involved - it's a slightly nebulous business; that's why they are being careful." Still, the team remains bullish about the remaining data analysis. "I'm not interested in being disappointed," says Francis Everitt of Stanford University, the project's principal investigator.

Meanwhile, the LAGEOS findings could be further improved before the end of the year by incorporating a new model of Earth's gravitational field, as gathered by the GRACE spacecraft. Erricos Pavlis of the University of Maryland in Baltimore County, who works with the LAGEOS data, says that it would be nice to beat GP-B, but even so he doesn't want to see it fail.

"After all these millions spent and decades of people's work," he says, "it's only fair that they get something out of this project." Sarah Tomlin

\section{Evidence for fourth neutrino fades}

A nine-year effort to resolve a mystery about the behaviour of neutrinos - particles that interact only weakly with matter - has thrown up an unexpected signal. The findings, announced on 11 April, leave open the possibility that new physics hides within the observations, but winnows the options for what that might be.

“The plot keeps thickening," says Bill Louis, co-spokesperson for the MiniBooNE neutrino detector based at Fermilab in Batavia, Illinois.

MiniBooNE aimed to settle controversy raised by the Liquid Scintillator Neutrino Detector (LSND) at Los Alamos National Laboratory in New Mexico, which looked at how one type of neutrino could turn into another, or 'oscillate. The LSND findings suggested that a fourth kind of neutrino existed - a sterile neutrino. But many scientists were sceptical of the result.

Using a 12-metre sphere filled with 800 tonnes of mineral oil to catch neutrinos, the MiniBooNE team found no evidence, at high neutrino energies, for the sort of oscillation that workers at LSND had reported (A. A. Aguilar-Arevalo et al. preprint at http://arxiv.org/abs/0704.1500; 2007). "My view is that there is no longer any credible evidence for sterile neutrinos," says Gary Feldman, a physicist at Harvard University. Team members say they are still working on the data and can't be so sure.

At low neutrino energies the detector saw more electron neutrinos in the experiment's beam of muon neutrinos than expected. The team can't yet explain the observation and the community's curiosity is piqued. "Already theorists are sending us their papers and saying look, we fit you," says project co-spokesperson Janet Conrad.

Meanwhile, MiniBooNE has switched to observing antineutrinos, which is what LSND studied, to find out whether the variations between the two experiments' results are due to surprising differences in the behaviour of matter and antimatter. Jenny Hogan 